\title{
Anti-N-Methyl-D-Aspartate Receptor Encephalitis with Concomitant Detection of Epstein-Barr Virus
}

\author{
Neuza Soares1, Emanuel Dias ${ }^{2}$, Mariana Formigo ${ }^{3}$, P. Torres-Ramalho4, Luis Braz ${ }^{5,6}$, \\ Reina-Couto Marta ${ }^{5,7}$ \\ ${ }^{1}$ Internal Medicine Department, Centro Hospitalar Universário de São João, Porto, Portugal \\ ${ }^{2}$ Gastroenterology Department, Centro Hospitalar Universitário de São João, Porto, Portugal \\ ${ }^{3}$ Internal Medicine Department, Hospital Senhora da Oliveira, Guimarães, Portugal \\ ${ }^{4}$ Intensive Medicine Department, Centro Hospitalar Universitário de São João, Porto, Portugal \\ ${ }^{5}$ Department of Neurology, Centro Hospitalar Universitário de São João, Porto, Portugal \\ ${ }^{6}$ Department of Clinical Neurosciences and Mental Health, Faculdade de Medicina da Universidade do Porto, Porto, Portugal \\ ${ }^{7}$ Pharmacology and Therapeutics Department, Faculdade de Medicina da Universidade do Porto, Porto, Portugal \\ Email: neuzasoares13@hotmail.com
}

How to cite this paper: Soares, N., Dias, E., Formigo, M., Torres-Ramalho, P., Braz, L. and Marta, R.-C. (2019) Anti-N-Methyl-DAspartate Receptor Encephalitis with Concomitant Detection of Epstein-Barr Virus. Neuroscience \& Medicine, 10, 194-198. https://doi.org/10.4236/nm.2019.103013

Received: June 15, 2019

Accepted: August 30, 2019

Published: September 2, 2019

Copyright $\odot 2019$ by author(s) and Scientific Research Publishing Inc. This work is licensed under the Creative Commons Attribution International License (CC BY 4.0). http://creativecommons.org/licenses/by/4.0/

\section{Open Access}

\begin{abstract}
Introduction: Anti-N-methyl-D-aspartate receptor (NMDAR) encephalitis is an important cause of encephalitis worldwide. While some cases are associated with neoplasms, in the remaining cases the etiology is unclear. Recent literature suggests that viral brain infections, mainly of the herpesviridae family, may be associated and/or trigger NMDAR encephalitis. Case Report: A 34-year-old woman with a 1-month-long history of progressive behavioral and language deterioration came to emergency department after a first unprovoked seizure followed by a focal status epilepticus. Brain computerized tomography was normal, but electroencephalography showed epileptiform activity over the left fronto-temporal region. She was admitted to the intensive care unit. Brain magnetic resonance imaging showed subtle $\mathrm{T} 2$ hypersignal in the parietal, fronto-opercular and insular regions. Cerebrospinal fluid (CSF) was positive for Epstein Barr virus (EBV) DNA whilst anti-NMDA antibodies were identified both in the CSF and blood. No tumors were detected after thorough investigation. Following intravenous steroids, plasma exchange and rituximab treatment she slowly improved being discharged home and at a 3-month follow-up she was sequels free. Conclusion: Despite the clear association between herpes virus simplex and NMDAR encephalitis, no such unequivocal relation has been reported for other virus, namely EBV. We report a case of NMDAR encephalitis which might be associated and triggered by EBV infection, identified by polymerase chain reaction.
\end{abstract}




\section{Keywords}

Autoimmune Encephalitis, Infectious Encephalitis, Herpesviridae Infections

\section{Introduction}

Anti- $N$-methyl- $D$-aspartate receptor (NMDAR) encephalitis is a newly recognized leading cause of encephalitis worldwide. It is characterized by subacute cognitive and behavioural symptoms, seizures, movement disorders and autonomic instability [1] [2]. In young females it is commonly a paraneoplastic manifestation of ovarian teratoma, but in more than half of the cases the etiology is unclear [2]. Recent clinical data suggests a strong relationship between herpes simplex (HSV) 1and encephalitis NMDAR encephalitis. The same connection has been suggested for other viruses, but the paucity of cases hinders this association [3].

We report a case of NMDAR encephalitis with Epstein Barr virus (EBV) identified by polymerase chain reaction (PCR).

\section{Case Report}

A 34-year-old woman patient with previously unremarkable medical history presented to the emergency department (ED) after a first unprovoked seizure followed by a focal status epilepticus. Her family reported progressive behavioral and language subtle changes over the previous month. In the ED, the patient had a brief complex motor seizure followed by subsequent generalized seizures interspersed with depressed consciousness. Electroencephalography (EEG) revealed several electrographic seizures in the left fronto-temporal region together with interspersed with continuous slow activity of the same area. Cerebrospinal fluid (CSF) showed 3 cells, normal proteins $(0.40 \mathrm{~g} / \mathrm{L})$ and normal glucose levels (73 mg/dL).

The patient was admitted in intensive care unit (ICU) medicated with levetiracetam and lacosamide leading to improvement of clinical seizures. Brain magnetic resonance imaging (MRI) showed subtle T2 hyperintensities and restricted diffusion involving left parietal, fronto-opercular and insular cortico-subcortical regions (Figure 1).

Cerebrospinal fluid EBV DNA revealed 900 copies per milliliter, but serum EBV IgM antibodies were negative. Other infectious agents were screened both in serum and CSF (syphilis, borrelia, herpes simplex, cytomegalovirus, varicella-zoster and human immunodeficiency virus, cryptococcus, mycobacteria) but were all negative. A comprehensive autoimmune panel revealed no abnormalities (anti-nuclear, anti-ds DNA, anti-cardiolipin, rheumatoid factor and anti-thyroid antibodies). Eleven days after admission, high titers of anti-NMDA antibodies were found both in CSF and blood, favoring the diagnosis of autoimmune encephalitis. Extensive paraneoplastic screening was performed and 


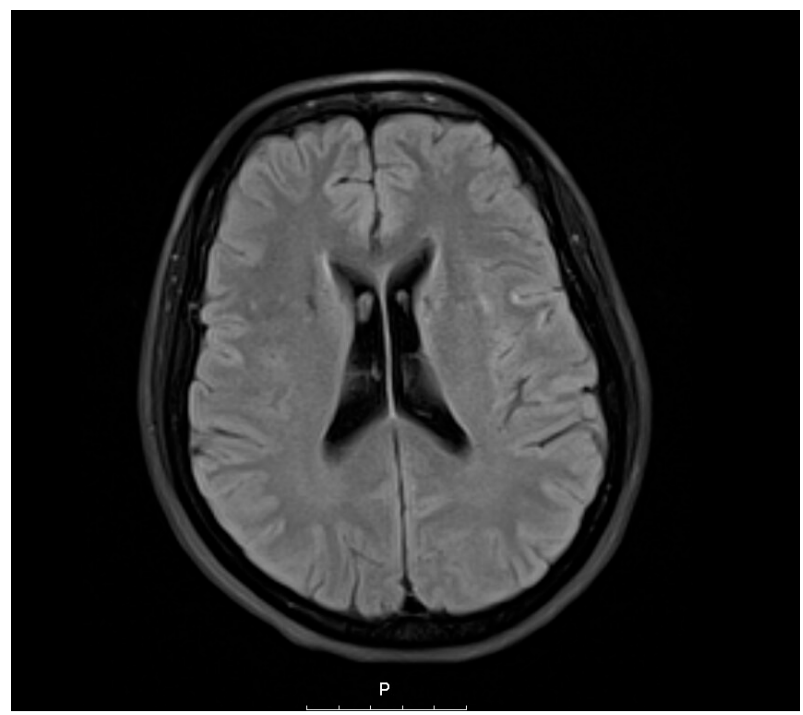

Figure 1. Axial T2-hipersinal involving left parietal, fronto-opercular and insular cortico-subcortical regions.

proved to be normal, incluind thoracoabdominopelvic computed tomography scanning, pelvic MRI, breast ultrasound, human papilloma virus, CA-125 and onconeuronal antibodies.

Clinically, the patient remained hemodynamic stable, expect for sinusal tachycardia and diaphoresis. Neurologically, she was quite slow with non-fluent speech, some periods of agitation and some episodes of epileptic fits. Convulsions were controlled with an increased dose of levetiracetam and lacosamide, subsequently and gradually discontinued. Repeated serial EEGs detected no epileptically activity, albeit slow and disorganizing activity, predominantly delta bush pattern with severe diffuse encephalopathy, compatible with NMDAR encephalitis.

Immunotherapy therapy was therefore started with a 5-day regimen of intravenous methylprednisolone followed six sessions of plasma exchange. After 17 days without any meaningful improvement, the patient was started on rituximab weekly for four weeks. Soon after the patient started to improve but kept some periods of periods of agitation and visual hallucinations. Later, she was transferred to the neurology ward and a month afterwards she was discharged home on the way to complete recovery and normalization of the EEG.

\section{Discussion}

We report a case that an EBV encephalitis, which may have been associated with/ortriggered the occurrence of NMDAR antibodies, compromising the course of the disease. Initially, when NMDAR encephalitis was first described by Dalmau et al. in 2007, it was typical of young females presenting with prominent changes of behaviour, psychosis, seizures, abnormal movements, autonomic dysfunction and coma in association with ovarian teratomas [3] [4]. Therefore, it was postulated that such tumors may exhibit nervous system-restricted anti- 
gens whose anti-tumor autoimmunity cross-reacting with the cognate brain protein, would be the underlying mechanism leading to the encephalitis [3]. Currently, present literature review still points to the female predominance of NMDAR encephalitis, although some $40 \%-80 \%$ of the patients carry no tumor at the time of diagnosis, with recent findings suggesting that viral brain infections may be another major cause for the development of autoimmune encephalitis [5] [6] [7].

The association of herpes simplex 1 (HSV) and NMDAR antibodies has been repeatedly documented. More recently, it has been suggested that other viruses such as varicella zoster, citomegalovirus, human herpes virus 6, enterovirus, adenovirus and human immunodeficiency virus, may also inducebrainstem encephalitis. However, much less is known about similar associations with these viruses and neurone surface antibodies and the role of EBV on this matter remains somewhat unclear [3] [4] [5] [8].

One case report has purposed EBV infection to be associated with anti-NMDA receptor encephalitis in spite of EBV DNA being absent in the CSF but, nevertheless, substantiated by positive IgM EBV antibodies in the blood [9]. Furthermore, data from the Mayo Clinic Virology and Neuroimmunology clinical laboratories points to the frequent coexistence of herpes viruses, namely EBV, and autoantibodies in patients with encephalitis (whether herpetic or autoimmune) inferring that the simultaneous presence of both, antoantibodies and herpes virus DNA, in encephalitis CSF may, in some patients, be responsible for the development of parainfections autoimmunity secondary to viral CNS infection (usually HSV encephalitis) [10].

Our case shows the association of EBV infection, confirmed by polymerase chain reaction (PCR) and NMDAR encephalitis, with no evidence of tumor as potential trigger. The pathophysiology of NMDAR encephalitis remains obscure. The herpes groups are neurotropic viruses with the potential to generate inflammatory responses, either upon limbic structures or in the brain stream, as in our case, leading to the release NMDAR epitopes into the immune system, breaking down tolerance and initiating autoimmune reactivity [4]. Mathew J. Kelly et al. have advanced that patients with bacterial meningitis have a very high prevalence of EBV in their serum and CSF with increased mortality. They propose that EBV plays a causative role and that EBV load is a better predictor of outcome rather than the sole presence of the virus per se. The resulting inflammation and the immune-mediated damage processes dictate the poor outcome [9]. Notably, in our patient, the autoantibodies and the virus genetic material were detectable at the beginning of the disease, suggesting that autoantibodies may also be an early event in viral encephalitis. This way, the viral destruction starts much earlier preceding clinical symptoms and allowing the immune system to mount an adequate antibody response. In our belief, another alternative might be that latent neurotropic virus may be reactivated as a consequence of CNS inflammation, and that the whole cascate of autoimmune mechanisms may then be triggered in response to the viral infection. 
In summary, that despite the clear association between HVS and NMDAR encephalitis, no such unequivocal relation has been reported for other viruses. We are reporting a case of NMDAR encephalitis which might have been triggered by EBV infection identified by PCR. That way, we can understand that EBV seems to be implicated in patients with anti-NMDA receptor antibody encephalitis but its role is elusive and it needs to be further evaluated.

\section{Conflicts of Interest}

The authors declare no conflicts of interest regarding the publication of this paper.

\section{References}

[1] Sutcu, M., et al. (2016) Role of Autoantibodies to N-Methyl-d-Aspartate (NMDA) Receptor in Relapsing Herpes Simplex Encephalitis: A Retrospective, One-Center Experience. Journal of Child Neurology, 31, 345-350. https://doi.org/10.1177/0883073815595079

[2] Venkatesan, A. (2013) Advances in Infectious Encephalitis: Etiologies, Outcomes, and Potential Links with Anti-NMDAR Encephalitis. Current Infectious Disease Reports, 15, 594-599. https://doi.org/10.1007/s11908-013-0382-9

[3] Pruss, H. (2017) Postviral Autoimmune Encephalitis: Manifestations in Children and Adults. Current Opinion in Neurology, 30, 327-333. https://doi.org/10.1097/WCO.0000000000000445

[4] Schabitz, W.R., et al. (2014) VZV Brainstem Encephalitis Triggers NMDA Receptor Immunoreaction. Neurology, 83, 2309-2311. https://doi.org/10.1212/WNL.0000000000001072

[5] Casares-Vivas, M., et al. (2016) Anti-NMDA Antibody Encephalitis Secondary to Herpes Simplex Virus Infection. Medicina Intensiva, 40, 193-195. https://doi.org/10.1016/j.medin.2015.06.004

[6] Guan, W., et al. (2015) Non-Tumor-Associated Anti-N-Methyl-D-Aspartate (NMDA) Receptor Encephalitis in Chinese Girls with Positive Anti-Thyroid Antibodies. Journal of Child Neurology, 30, 1582-1585. https://doi.org/10.1177/0883073815575365

[7] Zhang, W., Yan, L. and Jiao, J. (2017) Repeated Misdiagnosis of a Relapsed Atypical Anti-NMDA Receptor Encephalitis without an Associated Ovarian Teratoma. Neuroscience Letters, 638, 135-138. https://doi.org/10.1016/j.neulet.2016.11.057

[8] Solis, N., Salazar, L. and Hasbun, R. (2016) Anti-NMDA Receptor Antibody Encephalitis with Concomitant Detection of Varicella Zoster Virus. Journal of Clinical Virology, 83, 26-28. https://doi.org/10.1016/j.jcv.2016.08.292

[9] Xu, C.-L., et al. (2011) Anti-N-methyl-D-Aspartate Receptor Encephalitis with Serum Anti-Thyroid Antibodies and IgM Antibodies against Epstein-Barr Virus Viral Capsid Antigen: A Case Report and One Year Follow-Up. BMC Neurology, 11, 149. https://doi.org/10.1186/1471-2377-11-149

[10] Linnoila, J.J., et al. (2016) CSF Herpes Virus and Autoantibody Profiles in the Evaluation of Encephalitis. Neurology Neuroimmunology \& Neuroinflammation, 3, e245. https://doi.org/10.1212/NXI.0000000000000245 